\title{
The spectrum of $A T M$ missense variants and their contribution to contralateral breast cancer
}

\author{
Annegien Broeks · Linde M. Braaf • Angelina Huseinovic • \\ Marjanka K. Schmidt • Nicola S. Russell • \\ Flora E. van Leeuwen · Frans B. L. Hogervorst • \\ Laura J. Van 't Veer
}

Received: 2 February 2007/ Accepted: 12 February 2007/Published online: 28 March 2007

(C) Springer Science+Business Media B.V. 2007

\begin{abstract}
Heterozygous carriers of ATM mutations are at increased risk of breast cancer. In this case-control study, we evaluated the significance of germline ATM missense variants to the risk of contralateral breast cancer (CBC). We have determined the spectrum and frequency of ATM missense variants in 443 breast cancer patients diagnosed before age 50, including 247 patients who subsequently developed CBC. Twenty-one per cent of the women with unilateral breast cancer and $17 \%$ of the women with CBC had at least one ATM germline missense variant, indicating no significant difference in variant frequency between these two groups. We have found that carriers of an ATM missense mutation, who were treated with radiotherapy for the first breast tumour, developed their second tumour on average in a 92-month interval compared to a 136-month mean interval for those CBC patients who neither received RT nor carried a germline variant, $(p=0.029)$. Our results indicate that the presence of ATM variants does not have a
\end{abstract}

A. Broeks $(\bowtie) \cdot$ L. M. Braaf · A. Huseinovic .

M. K. Schmidt · L. J. Van 't Veer

Division of Experimental Therapy, The Netherlands Cancer

Institute, Plesmanlaan 121, 1066 CX Amsterdam,

The Netherlands

e-mail: abroeks@nki.nl

F. B. L. Hogervorst · L. J. Van 't Veer

Department of Pathology, The Netherlands Cancer Institute,

Amsterdam, The Netherlands

N. S. Russell

Department of Radiotherapy, The Netherlands Cancer Institute, Amsterdam, The Netherlands

F. E. van Leeuwen

Department of Epidemiology, The Netherlands Cancer Institute, Amsterdam, The Netherlands major impact on the overall risk of $\mathrm{CBC}$. However, the combination of RT and (certain) ATM missense variants seems to accelerate tumour development.

Keywords Breast cancer - ATM - Missense variants . Radiotherapy

\section{Introduction}

Homozygous or compound heterozygous germline mutations in the ATM gene cause the autosomal recessive disorder ataxia-telangiectasia (A-T). This progressive neurological childhood disease is characterized by cerebellar degeneration, immunological defects, extreme sensitivity for ionising radiation and increased risk for cancers, particularly lymphomas [1]. ATM mutations identified in A-T families can be classified in three categories; truncating mutations, mutations that lead to some expression of mutant protein that lacks kinase activity and missense mutations with reduced kinase activity (http:// chromium.liacs.nk/lovd/).

Heterozygous pathogenic ATM mutation carriers, $\sim 0.5-1 \%$ of the general population, do not display the symptoms observed in A-T patients. Several epidemiological studies have consistently shown elevated rates of breast cancer among female blood relatives of patients with A-T $[2,3]$. Thompson et al. have shown that the overall relative risk in carriers was 2.23 [95\% confidence interval (CI) 1.16-4.28] compared to the general population and 4.95 (95\% CI 1.9-12.9) in those younger that age 50. A large review showed that $A T M$ mutations are more frequent in breast cancer patients selected on the basis of a family history of breast cancer than in unselected patients [4]. Besides pathogenic ATM mutations, a large number of 
ATM variants (common polymorphisms and unclassified variants) have been described, which were found in cancer patients as well as in the general population. It has been hypothesized that the cancer risk among ATM heterozygotes might be related to mutation type, suggesting that particularly missense mutations are associated with an increased risk [5, 6]. However, two recent studies by Thompson et al. and Renwick et al. showed that pathogenic ATM mutations that cause A-T are breast cancer susceptibility alleles [2, 7]. This argues against the hypothesis that missense rather that truncating are associated with breast cancer.

Women with breast cancer have in general a three to fourfold increased risk of developing a new primary cancer in the opposite breast [8]. The contralateral breast cancer (CBC) risk might be explained by the same genetic and hormonal factors that caused the first breast cancer. Treatment related factors, e.g. radiotherapy for primary breast cancer, may also contribute to the development of cancer in the contralateral breast [9] (our own data, manuscript under review).

To evaluate whether germline ATM missense variants are significantly associated with $\mathrm{CBC}$ risk (results regarding ATM truncating mutations are reported elsewhere) and whether treatment modifies this risk, we conducted a case-control study in which we assessed the ATM missense mutation spectrum and frequency in women who developed their first breast cancer before age 50, with and without a second primary breast cancer.

\section{Methods}

\section{Patients}

The consecutive breast cancer patients included in this study were all selected from the hospital tumour registries of The Netherlands Cancer Institute, Amsterdam (NKI-AVL) or The Dr. Daniel den Hoed Cancer Center/ Erasmus Medical Center, Rotterdam (DDHK). Of all patients that were invited to participate we achieved an $80 \%$ response rate. The breast cancer patients were included if their (first) breast cancer was diagnosed before age $50 \quad(n=443)$. For $\mathrm{CBC}$ we required an interval of at least 1 year $(n=247)$. The unilateral breast cancer patients (UBC) patients all had to be disease-free (of a second breast cancer) for at least 5 years. The first $57 \mathrm{CBC}$ patients were individually age-matched (1:3) to UBC controls. All patients had invasive breast carcinoma and were treated with surgery. Of the CBC patients 169 did and 78 did not receive radiotherapy treatment for their primary breast tumour. Average age at diagnosis for the first breast cancer in the RT group was
41.2/41.3 years (mean/median) and the non-exposed group 42.0/43.2 years (mean/median). Detailed treatment data, disease and patient characteristics were obtained from medical records and risk factor questionnaires (data not shown) [10, 11]. Patients were asked to donate a $20 \mathrm{ml}$ blood sample or permission for use of paraffinembedded tissue blocks and patients gave their written informed consent for mutation analysis. This study received approval of the Medical Ethical Committees of NKI-AVL and DDHK.

\section{Genomic DNA isolation}

Genomic DNA was either isolated from peripheral blood lymphocytes with DNAzol (Invitrogen, Breda, The Netherlands) methods according to the manufacturer's instructions, or from three $10-\mu \mathrm{m}$ paraffin embedded normal tissue slides according to standard protocols [12]. For histopathological examination we used a hematoxylineosin stained slide.

\section{Mutation analysis}

The complete ATM Open Reading Frame (ORF) was analysed, each exon (exon 4-65) and all intron-exon boundaries were screened for germline mutations using Denaturing Gradient Gel Electrophoresis (DGGE) identifying $\sim 90 \%$ of all ATM mutations and polymorphisms (details from the author upon request). All aberrations were confirmed with genomic sequence analysis, performed using the ABI PRISM BigDyeTerminator Cycle Sequencing Ready Reaction Kit Version 3.1 (Applied Biosystems, Nieuwerkerk a/d yssel, The Netherlands). Sequencing products were analysed with the ABI PRISM 3700 DNA Analyzer and corresponding software.

\section{Statistical analysis}

Statistical analyses were performed using standard methods for analysis of case-control studies [13]. We compared the mutation frequency between UBC and CBC and between $\mathrm{CBC}$ cases previously treated with RT and cases not-treated with RT. Odds ratios (ORs) and 95\% CI were calculated to evaluate the association between mutation carriers status and breast cancer risk. We have used the Mann-Whitney test to determine whether the difference between the intervals between the two breast cancers of the CBC patients was significant. All analyses were performed using SPSS 12.0 (SPSS Inc., Chicago, IL, USA). 


\section{Results and discussion}

ATM germline mutations

In the present study, we have used the DGGE method to screen the complete ATM ORF to obtain insight in the ATM missense mutation spectrum in (contralateral) breast cancer patients. With DGGE we were able to confirm all the previously identified truncating mutations. A subset of the CBC patients described in this study had been screened in the past for ATM truncating mutations with the Protein Truncating Test, revealing seven ATM truncating mutations (including a non-sense mutation and small insertions and deletions; generating stop codons within a previously functional protein coding sequence causing premature termination of translation of the protein) [10]. Among all 443-breast cancer patients that were tested in this study with DGGE we detected a large number of ATM silent mutations (presumed neutral polymorphisms, data not shown and excluded from all analyses) and missense mutations (causing an amino acid substitution in the coded protein, most common ones; i.e. D1853N, not included in further analysis).

\section{ATM missense mutation spectrum}

In our study cohort we have detected 35 distinct ATM missense variants and 6 distinct truncating mutations. Several of the detected missense variants have been reported in the ATM database as being detected in A-T patients/or as polymorphisms (Table 1). None of the missense variants identified in this study are known as pathogenic A-T causing missense mutations. Seventeen of the missense variants have not been reported previously. Eleven missense variants were exclusively found in the CBC group and 10 exclusively in the UBC group. Whether this distinction in the spectrum indicates an association between particular variants and bilateral breast cancer risk cannot be concluded from the small numbers obtained in this study population. The ATM protein has several functional domains and the identified missense variants are located throughout the ORF. Potential functional implications of the newly identified unclassified variants remain to be established.

Despite the fact that $A T M$ plays a role in breast cancer risk, the role of most distinct ATM missense variants remains unclear. Some studies tried to predict the relevance of each particular mutation on basis of co-segregation with breast cancer in families, the location in a functional domain or interference with the splicing machinery. Only a few studies present functional analysis that are necessary to assess the biological impact of unidentified variants found frequently in ATM [18-20].
ATM missense mutations and contralateral breast cancer

Twenty-one per cent of the patients carried at least one ATM germline variant (missense and truncating; Table 2). Among the patients with CBC $(n=247)$ we identified in total 55 ATM variants in 45 individuals (18\%); 51 missense variants and 4 truncating mutations (Table 2). Eight CBC patients had multiple $A T M$ missense variants and 2 patients carried both a missense and a truncating ATM mutation. In the women with UBC $(n=196)$ we identified 52 ATM variants in 46 individuals (23\%); 48 missense and 4 truncating mutations. Three UBC patients carried double missense and 3 patients both a truncating and a missense variant. Although it is known from the literature that ATM missense variants might be involved in breast cancer pathogenesis, the identified $17 \%$ missense variant carriers among the CBC patients compared to the $21 \%$ missense variants among the UBC patients indicate that there is not a significantly increased risk for bilateral breast cancer among ATM missense variant carriers, OR 0.77 (95\% CI $0.48-1.24)$.

Association with radiation treatment

Women at high risk for developing breast cancer may respond differently to radiation exposures associated with screening and treatment, than the general population. Candidate-genes like ATM are implicated in maintenance of genome integrity. Their involvements in breast cancer susceptibility as well as their role in DNA-damage repair signalling make them excellent candidates for a role in radiation-induced breast cancer [21]. Recently, we showed that women with a pathogenic germline mutation in a DNA repair pathway gene (e.g. BRCA1, BRCA2, CHEK2 and $A T M$ ) have an over 2-fold increased risk of developing radiation-associated breast cancer (manuscript under review). Therefore, we now investigated whether exposure to ionising radiation had a greater biological impact on certain ATM genotypes than on others.

We did not detect a significantly increased risk of developing radiation-associated $\mathrm{CBC}$ among missense mutation carriers. Among those $169 \mathrm{CBC}$ patients who had developed a second primary breast tumour following radiotherapy for their first breast tumour we identified $19.5 \%$ ATM missense variants carriers compared to $13 \%$ among those CBC patients who did not receive RT, the OR from this case-only analysis is 1.65 [95\% CI $(0.77-3.55)$ $p=0.2]$. Furthermore, we have observed that $21 \%$ of the UBC patients, who received RT but did not develop a CBC carried an ATM missense variant, compared to $19.5 \%$ of the CBC patients that received RT for their first tumour [OR 0.86 (95\% CI 0.52-1.43)]. These results suggest that 
Table 1 ATM missense variant and truncating mutation spectrum in contralateral and unilateral breast cancer patients

\begin{tabular}{|c|c|c|c|c|}
\hline Missense variants & Amino acid change & $\mathrm{CBC} n=247$ & UBC $n=190$ & Database $^{\mathrm{a}}$ or literature \\
\hline $37 \mathrm{C}>\mathrm{T}$ & $\mathrm{R} 13 \mathrm{C}$ & & 1 & [10] \\
\hline $146 \mathrm{C}>\mathrm{G}$ & $\mathrm{S} 49 \mathrm{C}$ & 5 & 5 & database \\
\hline $162 \mathrm{~T}>\mathrm{C}$ & $\mathrm{Y} 54 \mathrm{H}$ & 2 & 1 & {$[4],[14]$} \\
\hline $378 \mathrm{~A}>\mathrm{T}$ & D126E & & 1 & database \\
\hline $1009 \mathrm{C}>\mathrm{T}$ & $\mathrm{R} 337 \mathrm{C}$ & & 1 & Novel \\
\hline $1132 \mathrm{~A}>\mathrm{G}$ & S377G & & 1 & Novel \\
\hline $1229 \mathrm{~T}>\mathrm{G}$ & V410A & 2 & 1 & {$[4]$} \\
\hline $1810 \mathrm{C}>\mathrm{T}$ & P604S & 1 & & database \\
\hline $2119 \mathrm{~T}>\mathrm{C}$ & S707P & 7 & 8 & database \\
\hline $2276 \mathrm{G}>\mathrm{A}$ & S759N & & & Novel \\
\hline $2336 \mathrm{~T}>\mathrm{C}$ & M779T & & 1 & Novel \\
\hline $2414 \mathrm{G}>\mathrm{A}$ & R805Q & 2 & & Novel \\
\hline $2572 \mathrm{~T}>\mathrm{C}$ & F858L & 4 & 3 & database \\
\hline $2650 \mathrm{C}>\mathrm{T}$ & $\mathrm{P} 884 \mathrm{~S}$ & 1 & & \\
\hline $2650 \mathrm{C}>\mathrm{T}$ & P884S & 1 & & Novel \\
\hline $2614 \mathrm{C}>\mathrm{T}$ & P872S & & & {$[15]$} \\
\hline $3161 \mathrm{C}>\mathrm{G}$ & P1054R & 8 & 13 & database \\
\hline $3925 \mathrm{G}>\mathrm{A}$ & A1309T & 1 & 1 & {$[16]$} \\
\hline $4138 \mathrm{C}>\mathrm{T}$ & H1380Y & 1 & & database \\
\hline $4258 \mathrm{C}>\mathrm{T}$ & L1420F & 5 & 4 & database \\
\hline $4324 \mathrm{~T}>\mathrm{C}$ & Y1442H & & 2 & Novel \\
\hline $4362 \mathrm{~A}>\mathrm{C}$ & K1454N & & 1 & database \\
\hline $4477 \mathrm{C}>\mathrm{G}$ & L1493V & 1 & & Novel \\
\hline $4664 \mathrm{~T}>\mathrm{A}$ & L1555H & & 1 & Novel \\
\hline $4722 \mathrm{G}>\mathrm{T}$ & L1574F & 1 & & Novel \\
\hline $5044 \mathrm{G}>\mathrm{T}$ & D1682Y & 1 & & database \\
\hline $5071 \mathrm{~A}>\mathrm{C}$ & S1691R & 2 & 2 & database \\
\hline $5557 G>A^{\mathrm{b}}$ & $D 1853 N$ & 35 & 49 & database \\
\hline $5558 \mathrm{~A}>\mathrm{T}$ & D1853V & 3 & 1 & database \\
\hline $5741 \mathrm{~A}>\mathrm{G}$ & D1914G & & & Novel \\
\hline $6067 \mathrm{G}>\mathrm{A}$ & G2023R & 1 & & database \\
\hline $6820 \mathrm{G}>\mathrm{A}$ & $\mathrm{A} 2274 \mathrm{~T}$ & 1 & & database \\
\hline $6919 \mathrm{C}>\mathrm{T}$ & L2307F & 1 & & [14] \\
\hline $7446 \mathrm{G}>\mathrm{A}$ & M2482I & 1 & & Novel \\
\hline $7874 \mathrm{~A}>\mathrm{G}$ & D2625G & & 1 & Novel \\
\hline $8659 \mathrm{C}>\mathrm{G}$ & H2887D & & 1 & Novel \\
\hline \multicolumn{5}{|c|}{ Truncating mutations } \\
\hline IVS10-6T >G & $419 X$ & 1 & 2 & database [10], [17] \\
\hline 1563delAG & $521 X$ & & 1 & database \\
\hline 1660delA & $554 X$ & 1 & & Novel \\
\hline $\mathrm{IVS} 14+2 \mathrm{~T}>\mathrm{G}$ & del 601-633 & 1 & & database \\
\hline 2572ins T & F858X & & 1 & Novel \\
\hline $3115 \mathrm{~A}>\mathrm{T}$ & R1039X & 1 & & Novel \\
\hline
\end{tabular}

a http://chromium.liacs.nk/lovd/

b Not included in frequency analysis 
Table $2 A T M$ variant frequencies in all breast cancer patients diagnosed under age 50 and according to uni- or contralateral breast cancer

\begin{tabular}{llll}
\hline Breast cancer patients with & All patients $n=443$ & CBC $n=247$ & UBC $n=196$ \\
\hline Total $A T M$ variants ${ }^{\mathrm{a}}$ & & $55: 51$ missense and 4 truncating & $52: 48$ missense and 4 truncating \\
At least one $A T M$ variant & $91(21 \%)$ & $45(18 \%)$ & $46(23 \%)$ \\
At least one $A T M$ missense variant & $85(19 \%)$ & $43(17 \%)$ & $42(21 \%)$ \\
Only one $A T M$ truncating mutations & 3 & 2 & 1 \\
One truncating and one missense variant & 5 & 2 & 3 \\
Double missense variants & 11 & 8 & 3 \\
\hline
\end{tabular}

a Not included are the most common and silent variants

$\mathrm{RT}$ is not a strong risk factor for the development of CBC among carriers of those ATM missense variants.

It has however been shown that particular alterations in the ATM gene are associated with increased radiation sensitivity [22-24]. Gutierrez-Enriquez et al. showed that lymfoblastoid cell lines carrying the ATM variant $3161 \mathrm{G}$ (linked to 2572C) was associated with increased in vitro chromosomal radio-sensitivity, perhaps by interfering with ATM function in a dominant-negative manner [22]. We found this particular variant allele (3161G/2572C) exclusively in our CBC group exposed to radiotherapy (four times) and not in the non-RT-exposed CBC group. This finding supports the hypothesis that particular ATM variants might play a differential role in radiation response. Although a subset of the missense variants was only detected in the RT exposed subpopulation, individual numbers were probably too small to detect a significant effect of particular mutations associated with treatment.

We observed that CBC patients with an ATM missense variant had an mean interval between the first and second breast tumour of $\sim 101$ months, compared to 122 months for non-carriers $\mathrm{CBC}$ patients $(p=0.085)$. Interestingly, the combination of radiation treatment and a missense variant resulted in an even shorter mean interval of a 92 months in the CBC patients compared to a 136-month interval for $\mathrm{CBC}$ patients who neither received RT nor carried a germline variant $(p=0.029)$. These data suggest that carrier-ship of an ATM missense variant may accelerate the development of a second tumour and decreases the age at onset of the second breast tumour, especially in case of exposure to RT.

The suggestion of a shorter induction period of RT-associated breast cancer in patients, who carry an ATM missense mutation, while the proportion of patients with missense variants was similar in $\mathrm{CBC}$ and UBC cases, might be attributable to a different spectrum of mutations in those patients who developed CBC. A big challenge in such a study remains to assess which particular missense mutations have an impact on ATM function. Large association studies, as performed by the Breast Cancer Association Consortium (coordinated by Doug Easton and
Paul Pharoah, Cambridge), and functional studies are clearly necessary to determine the importance of particular variants and their contribution to the breast cancer risk.

Acknowledgements We would like to thank Jos Urbanus and Geert van Haalem for performing mutation screens. We would like to thank Prof. Dr. Jan Klijn for contributing patients from the DDHK. Funding of this study was provided by the Dutch Cancer Society (DCS-NKI 01-2425).

\section{References}

1. Taylor AM, Harnden DG, Arlett CF et al (1975) Ataxia telangiectasia: a human mutation with abnormal radiation sensitivity. Nature 258:427-429

2. Thompson D, Duedal S, Kirner J et al (2005) Cancer risks and mortality in heterozygous ATM mutation carriers. J Natl Cancer Inst 97:813-822

3. Cavaciuti E, Lauge A, Janin N et al (2005) Cancer risk according to type and location of ATM mutation in ataxia-telangiectasia families. Genes Chromosomes Cancer 42:1-9

4. Thorstenson YR, Roxas A, Kroiss R et al (2003) Contributions of ATM mutations to familial breast and ovarian cancer. Cancer Res 63:3325-3333

5. Gatti RA, Tward A, Concannon P (1999) Cancer risk in ATM heterozygotes: a model of phenotypic and mechanistic differences between missense and truncating mutations. Mol Genet Metab 68:419-423

6. Chenevix-Trench G, Spurdle AB, Gatei M et al (2002) Dominant negative ATM mutations in breast cancer families. J Natl Cancer Inst 94:205-215

7. Renwick A, Thompson D, Seal S et al (2006) ATM mutations that cause ataxia-telangiectasia are breast cancer susceptibility alleles. Nat Genet 38:873-875

8. Harvey EB, Brinton LA (1985) Second cancer following cancer of the breast in Conneticut, 1935-1982. J Natl Cancer Inst Monogr 68:99

9. Boice JD Jr, Harvey EB, Blettner M et al (1992) Cancer in the contralateral breast after radiotherapy for breast cancer. N Engl J Med 326:1357-1358

10. Broeks A, Floore AN, Urbanus JHM et al (2000) ATM germline mutations do contribute to breast cancer susceptibility. Am J Hum Genet 66(2):494-500

11. Broeks A, de Witte L, Nooijen A et al (2004) Excess risk for contralateral breast cancer in CHEK $2 * 1100 \mathrm{delC}$ germline mutation carriers. Breast Cancer Res Treat 83:91-93

12. Sambrook J, Fritsch EF, Maniatis T (1989) Molecular cloning: a laboratory manuel, 2nd edn. Cold Spring Harbor Laboratory Press, Cold Spring Harbor, New York 
13. Breslow NE, Day NE (1980) Statistical methods in cancer research. In: Davis W (ed) International agency for research on cancer. IARC Scientific Publications, Lyon, France

14. Yuille MR, Condie A, Hudson CD et al (2002) ATM mutations are rare in familial chronic lymphocytic leukemia. Blood 100:603-609

15. Bretsky P, Haiman CA, Gilad S et al (2003) The relationship between twenty missense ATM variants and breast cancer risk: the multiethnic cohort. Cancer Epidemiol Biomarkers Prev 12:733-738

16. Greiner TC, Dasgupta C, Ho VV et al (2006) Mutation and genomic deletion status of ataxia telangiectasia mutated (ATM) and p53 confer specific gene expression profiles in mantle cell lymphoma. Proc Natl Acad Sci USA 103:2352-2357

17. Bernstein JL, Teraoka S, Southey MC et al (2006) Populationbased estimates of breast cancer risks associated with ATM gene variants c.7271T $>\mathrm{G}$ and c.1066-6T $>\mathrm{G}$ (IVS10-6T $>\mathrm{G}$ ) from the breast cancer family registry. Hum Mutat 27:1122-1128

18. Thorstenson YR, Shen P, Tusher VG et al (2001) Global analysis of ATM polymorphism reveals significant functional constraint. Am J Hum Genet 69:396-412
19. Scott SP, Bendix R, Chen P et al (2002) Missense mutations but not allelic variants alter the function of ATM by dominant interference in patients with breast cancer. Proc Natl Acad Sci USA 99:925-930

20. Lavin MF, Scott S, Gueven N et al (2004) Functional consequences of sequence alterations in the ATM gene. DNA Repair (Amst) 3:1197-1205

21. Bennett LM (1999) Breast cancer: genetic predisposition an exposure to radiation. Mol Carcinog 26:143-149

22. Gutierrez-Enriquez S, Fernet M, Dork T et al (2004) Functional consequences of ATM sequence variants for chromosomal radiosensitivity. Genes Chromosomes Cancer 40:109-119

23. Smilenov LB, Brenner DJ, Hall EJ (2001) Modest increased sensitivity to radiation oncogenesis in ATM heterozygous versus wild-type mammalian cells. Cancer Res 61:5710-5713

24. Angele S, Romestaing P, Moullan N et al (2003) ATM haplotypes and cellular response to DNA damage: association with breast cancer risk and clinical radiosensitivity. Cancer Res 63:8717-8725 